Proc. Indian Acad. Sci. (Earth Planet. Sci.). Vol. 92. Number 2, July 1983, pp. 165-177.

- Printed in India.

\title{
Very low frequency electromagnetic prospecting method - A few field applications in India
}

\author{
I B RAMA PRASADA RAO, R NAGENDRA, \\ S L NARASIMHA RAO and J VENKATRAMAIAH \\ Centre of Exploration Geophysics, Osmania University, Hyderabad 500 007, India
}

MS received 9 December 1981: revised 19 January 1983

\begin{abstract}
The very low frequency electromagnetic prospecting equipment measures different space and phase components of the electromagnetic field in the frequency range $15-25 \mathrm{kH}$ / emanating from distant naval radio stations. The field application of the equipment designed and constructed for this purpose has demonstrated the usefulness of the investigations in groundwater and geological mapping. The amplitude measurements necessitate correction of the diurnal variations of the electromagnetic field at the observation point.
\end{abstract}

Keywords. Elliptic polarization; diurnal variation; groundwater investigations; granitic terrain; very low frequency electromagnetic equipment.

\section{Introduction}

Very low frequency electromagnuiic (VI F PM) method (Sedelnikov et al 1971; Paterson and Ronka 1971) utilises electromagnetic fields in the $\iota$ ange 15 to $25 \mathrm{kHz}$ radiated by powerful naval radio stations. At sufficiently large distances from the transmitting antenna (distance $R \gg$ wavelength $\lambda$ ) the electromagnetic field near the homogeneous earth can be approximated to a plane wave with the magnetic field horizontal at right angles to the radius vector connecting the transmitting station to the observation point (figure 1). Whenever a conductive geological body, whose strike direction is nearly parallel to the propagation path, is present in an otherwise homogeneous earth, eddy currents are induced in the body giving rise to a secondary magnetic field. This secondary magnetic field will have the same frequency as that of the primary field but lesser in amplitude and differs in direction and phase. The phase of the secondary field will be between $\pi / 2$ and $\pi$ depending upon the product $|K d|$ where $K$ is the wavenumber given by $K=\left(\epsilon \mu \omega^{2}+j \sigma \mu \omega\right)^{1 / 2}$ and $d$ is the characteristic linear dimension of the geological body*. At the observation point, the total magnetic field is elliptically polarized in general.

In VLF prospecting it is the general practice to determine either (a) the different space components of the total magnetic field and the tilt $\theta$ (the inclination of the major axis of the ellipse of polarization with the horizontal) in degrees or the tangent of the tilt in per cent or (b) the ellipticity $\rightarrow$ which is the ratio of the major to minor axes and the tilt $\theta$ or (c) the magnetic field intensity in the direction perpendicular to the radius vector $(R)$ and the electrical field component in the earth (by means of grounded electrodes separated by some distance) in the direction of the radius vector, such that the appa rent resistivity $\rho_{a}$ can be computed using the relation

\footnotetext{
* $a$ is the conductivity $(\mathrm{ohm} / \mathrm{m}) ; \mu$ is the magnetic permeability (Henry/m); $\epsilon$ is the dielectric constant $($ Farad $/ \mathrm{m})$ and $\omega$ is the angular frequency (Radians/ $: z_{c}$.).
} 


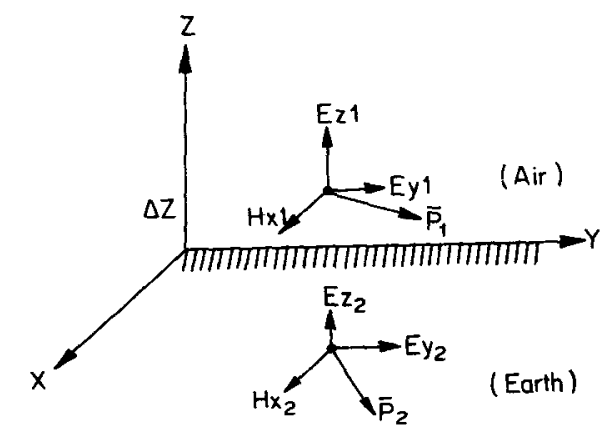

Figure 1. Propagation of VI.FFM field.

$$
\rho_{a}=\frac{1}{\omega \mu}\left|\frac{E_{Y}}{H_{X}}\right|^{2}
$$

where $E_{Y}$ and $H_{X}$ are the horizontal electric and magnetic field components on the earth's surface respectively.

The VI.F method enjoyed popularity all over the world due to the speed of field operation and low overall operation cost. Further. from the existing theory of the EM method and field application in general the following advantages can be attributed to the VI.F EM technique.

(a) The depth of penetration is fairly large $(20-50 \mathrm{~m}$ for ground resistivities of the order of few hundreds of ohm-m) when compared to those methods which make use of frequencies in the range of $150 \mathrm{kHz}$ to few $\mathrm{MHz}$.

(b) Owing to the relatively higher frequencies employed when compared to the other EM methods such as SLINGRAM, TURAM, etc., relatively poorer conductors will also contribute to the signal. As a result this technique can be employed in ground water investigations in hard rock terrain. geological mapping and fresh/ salt water interface studies, etc.

(c) The VIFEM field attenuates slowly with distance from radio stations and are relatively stable in intensities.

However, application of this method in prospecting for very good conductors like polymetallic deposits may pose a problem in view of the fact that one has to identify the required 'signal' from among several anomalies caused by different geological factors in the region.

In India. so far the potential of this technique has not been utilised in groundwater prospecting for rapid coverage of drought prone areas. Instead quite often time consuming electrical resistivity methods are being utilised extensively as the primary methods. Geological mapping is another a rea where the capabilities of this method are not being fully exploited in India.

This paper is intended to demonstrate the application of the VLF EM equipment designed and constructed in the Department of Geophysics, Osmania University, in geological mapping and ground water investigations in hard rock terrain by presenting a few field studies in India. A brief theory. equipmental details. field procedure adopted and the detailed discussion of the field results obtained are presented in this paper. 


\section{Theoretical basis}

The EM field of the radio antenna is equivalent to the field produced by a vertical electrical dipole placed on the earth's surface.

Let the transmitting electrical antenna of length $\Delta Z$ carrying an alternating current $I$ with a circular frequency $\omega$ be placed at the origin of the spherical coordinate system $R, \theta, \varphi$. Let the propagation constant for air $K_{0} \approx \omega\left(\epsilon_{0} \mu_{0}\right)^{1 / 2}$ and for the homogeneous conductive earth $K=\left(\epsilon \omega^{2} \mu+j \sigma \mu \omega\right)^{1 / 2}$. At distances $R \gg \lambda_{0}\left(\lambda_{0}\right.$ is the wavelength in air), the electric field may be expressed as (Stratton 1941)

$$
E=E_{1}+E_{2}
$$

where

$$
\begin{aligned}
E_{1}= & -\left(j \omega \mu_{0} / 4 \pi\right) I \Delta z \sin \theta\left(1+R_{1}\right)[\exp (j K R) / R] a_{\theta} \\
E_{2}= & \left(j \omega \mu_{0} / 4 \pi\right) I \Delta z\left(1-R_{1}\right) F[\exp (j K R) / R] \times \\
& \times\left[a_{z}+a_{i}\left(\sin \theta / Z_{21}^{2}\right)\left(1+0.5 \cos ^{2} \theta\right)\left(Z_{21}^{2}-\sin ^{2} \theta\right)^{1 / 2}\right. \\
R_{3}= & \frac{Z_{21}^{2} \cos \theta-\left(Z_{21}^{2}-\sin ^{2} \theta\right)^{1 / 2}}{Z_{21}^{2} \cos \theta+\left(Z_{21}^{2}-\sin ^{2} \theta\right)^{1 / 2}} \\
F= & {[1+j \sqrt{\pi W} \exp (-W) \operatorname{erfc}(-j \sqrt{W})] } \\
\operatorname{erf} \cdot(- & j \sqrt{W})=2 / \sqrt{\pi} \int_{-j}^{\infty} \exp \left(-x^{2}\right) \mathrm{d} x \\
W= & 0.5 j K R\left[Z_{21}^{2} \cos \theta+\left(Z_{21}^{2}-\sin ^{2} \theta\right)^{1 / 2}\right]^{2} / Z_{21}^{4} \\
Z_{i 1}^{2}= & \frac{\epsilon}{\epsilon}\left(1+\frac{j \sigma}{\omega \epsilon}\right)
\end{aligned}
$$

$a_{A}$ is a unit vector perpendicular to the radius vector $R$, while $a_{z}$ and $a_{r}$ are the unit vectors in a cylindrical coordinate system parallel to the $Z$ and $R$ directions.

It can be shown that, if the conductivity of the earth is finite $R_{\mathrm{r}} \rightarrow-1$ for $\theta \rightarrow \pi / 2$ and then $E_{1} \rightarrow 0$. In such a case only the $E_{2}$ component exists and is termed as the ground wave. Converting into the Descartes coordinate system, it can be shown that only one horizontal magnetic component $H_{X}$ remains near the earth's surface given by

$$
H_{x}=-\sqrt{\epsilon^{\prime} / \mu} E_{1}
$$

where 


$$
\epsilon^{\prime}=\epsilon+j \sigma / \omega
$$

Thus on measuring the surface impedance $|Z|=\left|H_{X} / E_{Y}\right|$ and neglecting the influence of the displacement currents the resistivity $\rho$ can be determined.

Further whenever a local conductor is present in the homogeneous earth, the magnetic field is elliptically polarized.

\section{Elliptical polarization}

Let the linearly polarized primary and secondary magnetic fields be represented by $H_{p}=H_{p 0} \cos \omega t$ and $H_{s}=H_{s(1} \cos (\omega t-\varphi)$ respectively where $\phi$ is the phase difference between $H_{p}$ and $H_{s}$. Further, let the space angle between $H_{p}$ and $H_{s}$ be $\alpha$. Let the space coordinate system $\mathrm{X}, \mathrm{Y}, \mathrm{Z}$ be oriented such that the primary field $H_{p}$ is along the $\mathrm{OX}$ axis and the direction of propagation is along the $\mathrm{Y}$ axis (figure 2) and the $\mathrm{Z}$ axis is oriented along the vertical.

Following Svetov (1973) different components of the total magnetic field ( $H_{T}=$ $H_{p}+H_{s}$ ) which is elliptically polarized are given by the horizontal component of the total field

$$
H_{T X}=\left(H_{p 0}^{2}+H_{s 0}^{2} \cos ^{2} \alpha+2 H_{p 1} H_{s 0} \cos \alpha \cos \varphi\right)^{1 / 2}
$$

Vertical component of the total field

$$
H_{T Z}=H_{s 0} \sin \alpha
$$

Semi major axis

$$
\begin{aligned}
H_{a}= & 0.5\left[H_{p 0}^{2}+H_{s 0}^{2}+2 H_{p 0} H_{s 0} \cos (\alpha-\varphi)\right]^{1 / 2}+ \\
& +\left[H_{p 0}^{2}+H_{s 0}^{2}+2 H_{p 0} H_{s 0} \cos (\alpha+\varphi)\right]^{1 / 2}
\end{aligned}
$$

Semi minor axis

$$
\begin{aligned}
H_{h}= & 0.5\left[H_{n 0}^{2}+H_{s 0}^{2}+2 H_{p 0} H_{s 0} \cos (\alpha-\varphi)\right]^{12}- \\
& -\left[H_{p 0}^{2}+H_{s 0}^{2}+2 H_{p 0} H_{s 0} \cos (\alpha+\varphi)\right]^{1 / 2}
\end{aligned}
$$

usually $H_{s} \ll H_{p}$ and hence

$$
\begin{aligned}
& H_{a} / H_{p} \approx 1+\left(H_{s} / H_{p}\right) \cos \varphi \cdot \cos \alpha=1+\left(\operatorname{Re} H_{s x} / H_{p}\right) \\
& H_{h} / H_{p} \approx\left(H_{p} / H_{n}\right) \sin \varphi \cdot \sin \alpha=\operatorname{Im} H_{s z} / H_{r}
\end{aligned}
$$

tilt of the major axis

$$
\boldsymbol{\theta} \approx \boldsymbol{H}_{s} \cos \varphi \cdot \sin \alpha / \boldsymbol{H}_{p}=\operatorname{Re} \boldsymbol{H}_{s=} / \boldsymbol{H}_{r}
$$




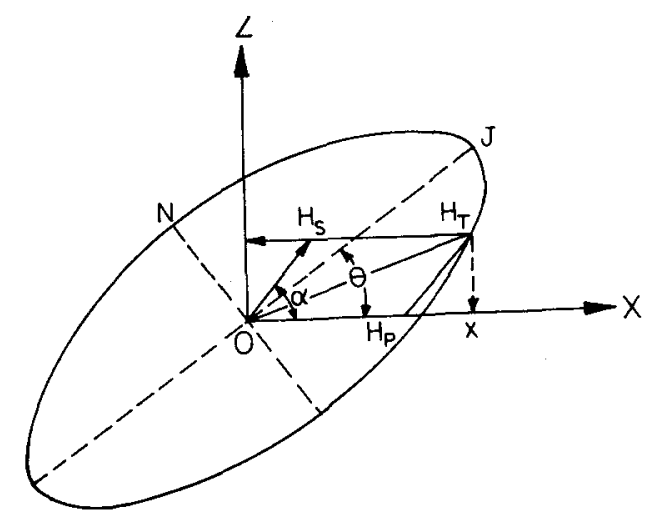

Figure 2. Ellipse of polarization.

From the above relations, it is evident that the profiling curves of the-semi major ax is are similar to the horizontal component (parallel to the primary field) of the secondary field while its frequency characteristics are similar to the real part of the horizontal component of the secondary field. On the other hand, the profiling curves of the semi minor axis are similar to the vertical component of the secondary field while its frequency characteristics are similar to that of the imaginary part of the vertical component of the secondary field. The tilt angle of the major ax is behaves similarly as the real part of the vertical component of the secondary field. Thus by measuring. either the components of the total magnetic field or the pola rization ellipse parameters (which are in turn related to the secondary field) it is possible to obtain the geometric and electrical parameters of the local geological objects.

\section{Interpretation of VIF EM data}

Quantitative interpretation of the VLF EM data is complicated and is not fully developed. However. Fraser (1969) suggested a filter for contouring the VLF tilt angle measurements data which helps in reducing the noise and transforming the true cross-overs to positive peaks. Paterson and Ronka (1971) suggested thumb rules to calculate the depth to the top of the conductor from the distance between the peaks of the real conponent profiles. For near surface conductors. the depth is over estimated by the thumb rule (Coney 1977). Baker and Myers (1979) conducted modelling investigations on the sheet response due to conductors in a conducting medium and developed interpretation curves. Kaikkonen (1977), Olsson (1980). Sacit Saydam (1981) and Poddar (1981. 1982) presented interpretational schemes for the half plane conductors embedded in a conductive environment. Baker and Myers (1980) and Eberle (1981) presented terrain correction for VLF EM data. Based on the characteristic points, Tarkhov (1961) reported simple relations for determining the depth to vertical conductive vein type bodies and contact between two media differing in electrical parameters.

\section{Equipment}

The tangent of the tilt of the major axis from the horizontal in per cent (termed as real) and the ellipticity (termed as imaginary or quadrature) in per cent is measured by 


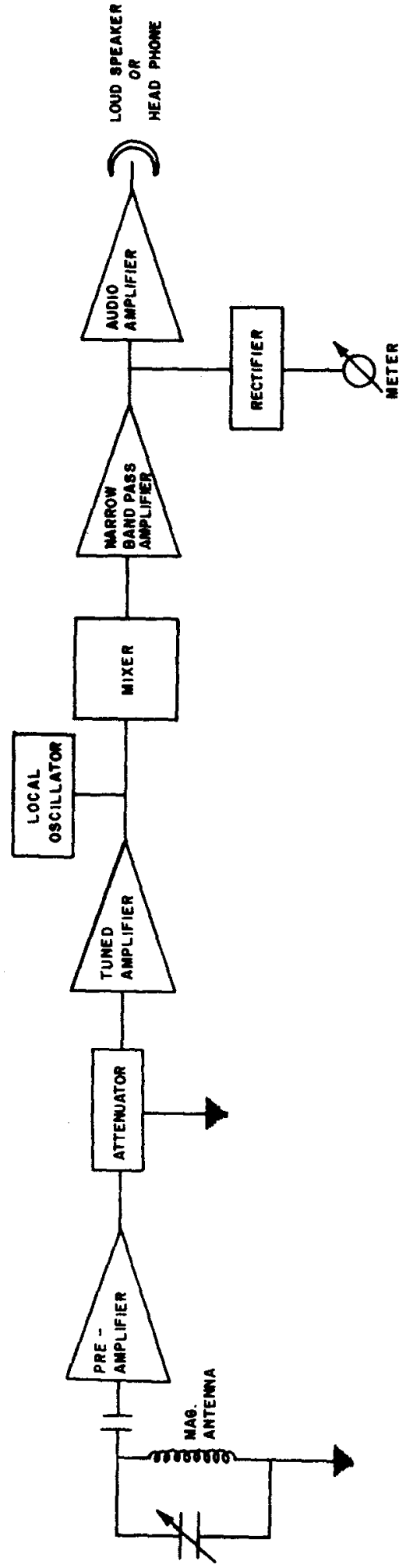

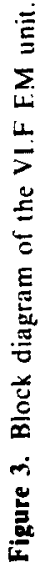


EM 16 (Geonics) system while EM 16 R (Geonics) measures the impedance of the VIFFM field on the earth's surface. Phoenex system measures the orientation and magnitudes of the major and minor axes of the polarization ellipse. Radem system is employed for measuring the tilt of the major axis in degrees and the ellipticity in per cent. The Scint rex VI.F EM equipment measures the amplitudes and geometry parameters of the polarization ellipse. Soviet made VIF EM equipment measures the space components of the total field and the tilt of the major ax is of the ellipse of polarization.

The equipment designed by the authors is based on superheterodyne receiver system and is capable of measuring the VL.F field emanating from NWC-Australia $(22.3 \mathrm{kHz}$ ). The block diagram of the equipment is presented in figure 3 and the general view of the entire equipment is presented in figure 4.

The magnetic receiving antenna consists of a ferrite core of $20 \mathrm{cms}$ containing six sections of coils with a sensitivity of $25 \mathrm{Vm} / \mathrm{A}$ at $22.3 \mathrm{kHz}$ which can be tuned with the help of a variable condenser provided on the front panel of the equipment. This antenna is rotatable around the vertical and horizontal axes for measuring different space components and the tilt. The signal received by the antenna is fed to a preamplifier stage having a gain of 40 designed on FETBFW-11. The output of the amplifier is fed to an attenuator which is incorporated in the emitter circuit of the emitter repeater and provides 30,100 and $300 \mu \mathrm{V}$ full scale ranges for the equipment. The output of the emitter repeater is fed to a tuned amplifier which is designed on IC-741, and has a gain of 50. The local oscillator constructed on LC circuit has a frequency of $26.4 \mathrm{k} \mathrm{Hz}$. The

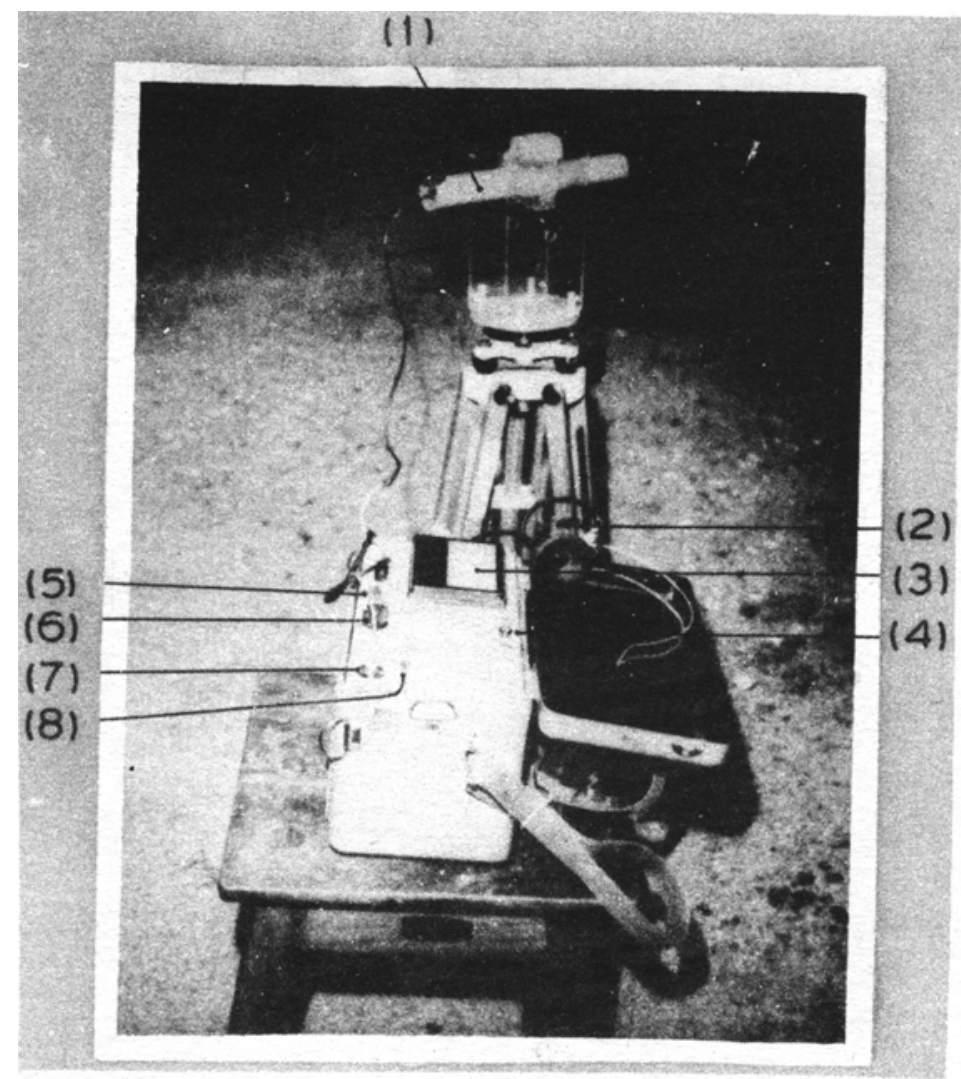

Figure 4. VLF EM equipment. 
frequency of the oscillator is stable to 0.1 in $10^{3}$ for a continuous operation of $3 \mathrm{hrs}$. The output of the local oscillator and the tuned amplifier are mixed in a mixer stage constructed on a diode whose load is an LC circuit tuned to $4.1 \mathrm{kHz}$. (intermediate frequency). The output of the mixer is fed to an if tuned amplifier with a gain of 20 having a $Q$ of 3 at IF $4.1 \mathrm{kHz}$. The signal from the IF amplifier is fed to a fullwave rectifier and then measured by a panel meter. The output of the tuned amplifier is also fed to an audio amplifier, the output of which is given to the head phone.

For measuring the magnetic field components, the magnetic antenna is connected to the input and when the impedance measurements are to be made the grounded electric dipole of length $50 \mathrm{~m}$ is connected to the input after measuring the magnetic field component. The selection of the antenna is made by a toggle switch provided on the front panel of the equipment.

\section{Field procedure}

At every observation point at first. the axis of the magnetic antenna is kept horizontal and rotated around a vertical axis for maximum pick up. While measuring the magnetic field components. the magnetic antenna is tuned with the help of a tuning condenser before taking the observation. The maximum pick up is indicated by a clear loud signal in the earphone and a maximum reading in the panel meter. which is noted down and is termed as $H \rho_{\max }$.

To measure the tilt. the magnetic antenna is rotated around a vertical axis for minimum pick up and then lifted in a vertical plane (rotated around a horizontal axis) for a further minimum signal. The tilt angle is noted from a protractor scale (not shown in figure 4).

For measuring the vertical component $H$ : the magnetic antenna is kept vertical.

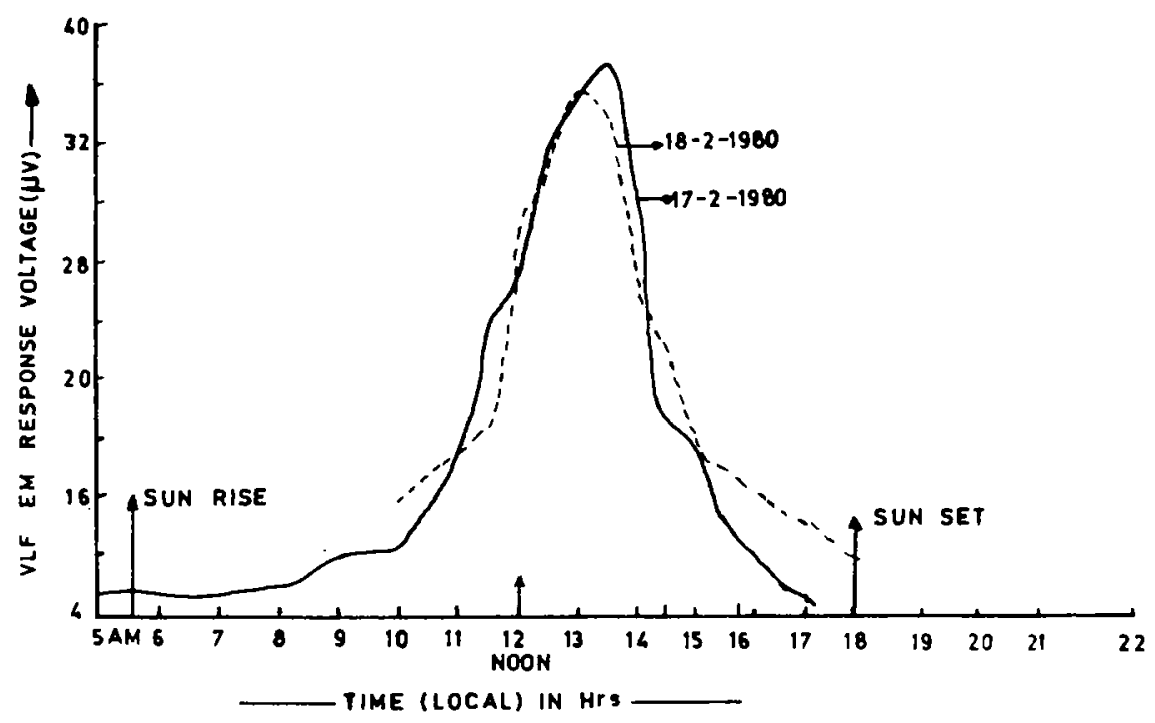

Figure 5. Diurnal variation of VI FFM field from VWC. Australia. 
Since we a re interested in relative variations of the signal from the base point along a profile, the profiling data in which $H \rho_{\max }$ and $H_{2}$ are measured can be expressed either in $\mu \mathrm{V}$ or $\mathrm{A} / \mathrm{m}$ (using a sensitivity value of $25 \mathrm{Vm} / \mathrm{A}$ for the magnetic antenna). However, while taking the impedance measurements for computing $\rho_{a}$ values from (1), the electric component $E$ is expressed in $\mathrm{V} / \mathrm{m}$ and the magnetic component is expressed in $\mathrm{A} / \mathrm{m}$ so that the impedance $Z$ has the dimension of ohm.

In practice the intensity measurements have diurnal variations similar to that observed in magnetic methods of prospecting. Figure 5 gives the diurnal variations of $H \rho_{\max }$ observed continuously for two days. It can be noted that the intensity considerably increases from $0930 \mathrm{hrs}-1230 \mathrm{hrs}$ local time after which it decreases. For field observations made between $0930 \mathrm{hrs}$ and $1730 \mathrm{hrs}$ local time the diurnal corrections are to be applied by occupying the base station(s) as in the magnetic methods of prospecting and corrected for the variation of intensity with time.

\section{Field applications in India}

The apparent resistivity $\rho_{a}$ values computed from (1) from the surface impedance measurements are shown in figure 6. During these measurements the electric component was measured using a elect ric dipole of length $50 \mathrm{~m}$. Symmetrical Schlumberger resistivity data with a current electrode separation of $10 \mathrm{~m}$ is also shown in figure 6 . Both the results show a lowering in resistivity between $80-140 \mathrm{~m}$ stations. However. this zone is more prominent and localized in the $\rho_{a}$ value computed from the VLFEM data. Investigations by Schlumberger electrical soundings and borewell (for tapping groundwater) placed in the low resistivity zone revealed a water bearing weathered granite (Patangay 1977).

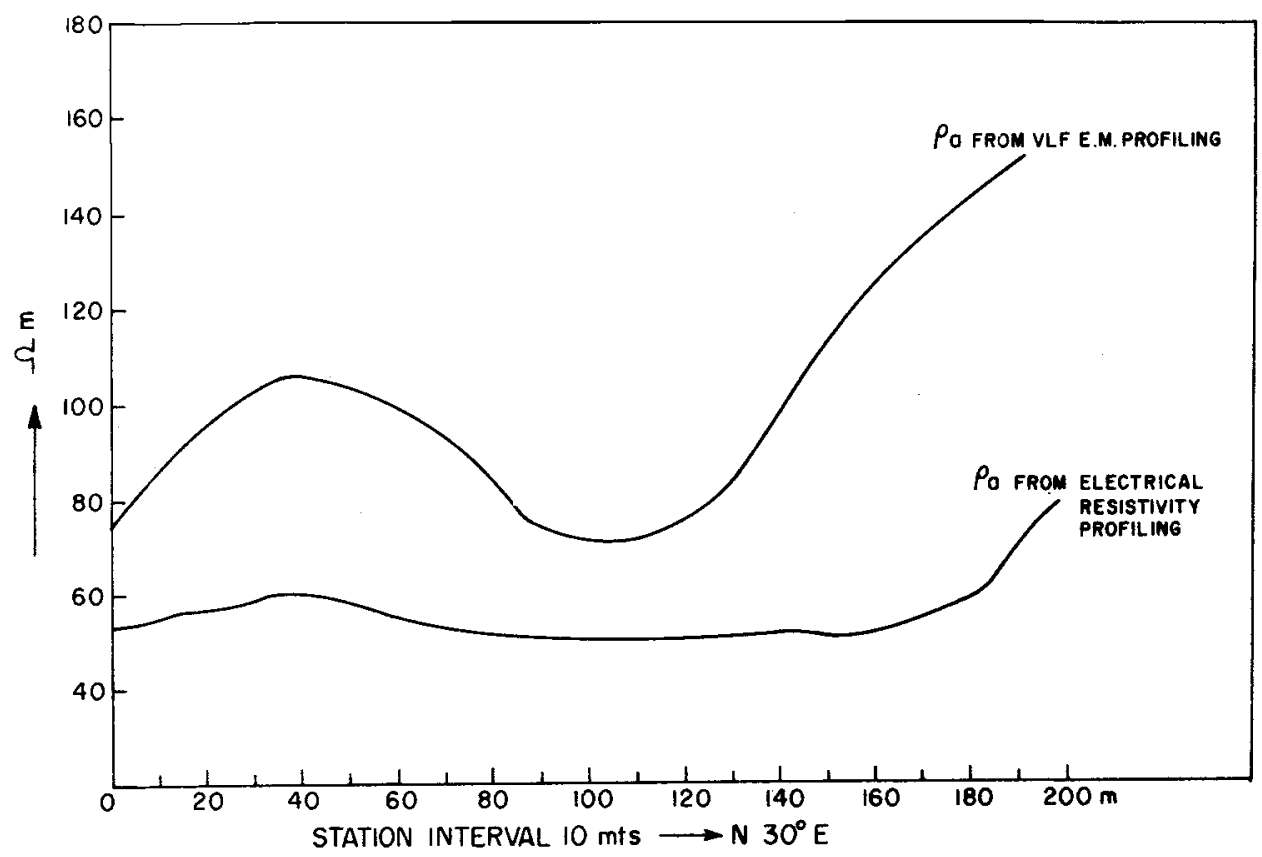

Figure 6. VLFEM and Schlumberger $(A B / 2=10 \mathrm{~m})$ profiling in groundwater investigations. 


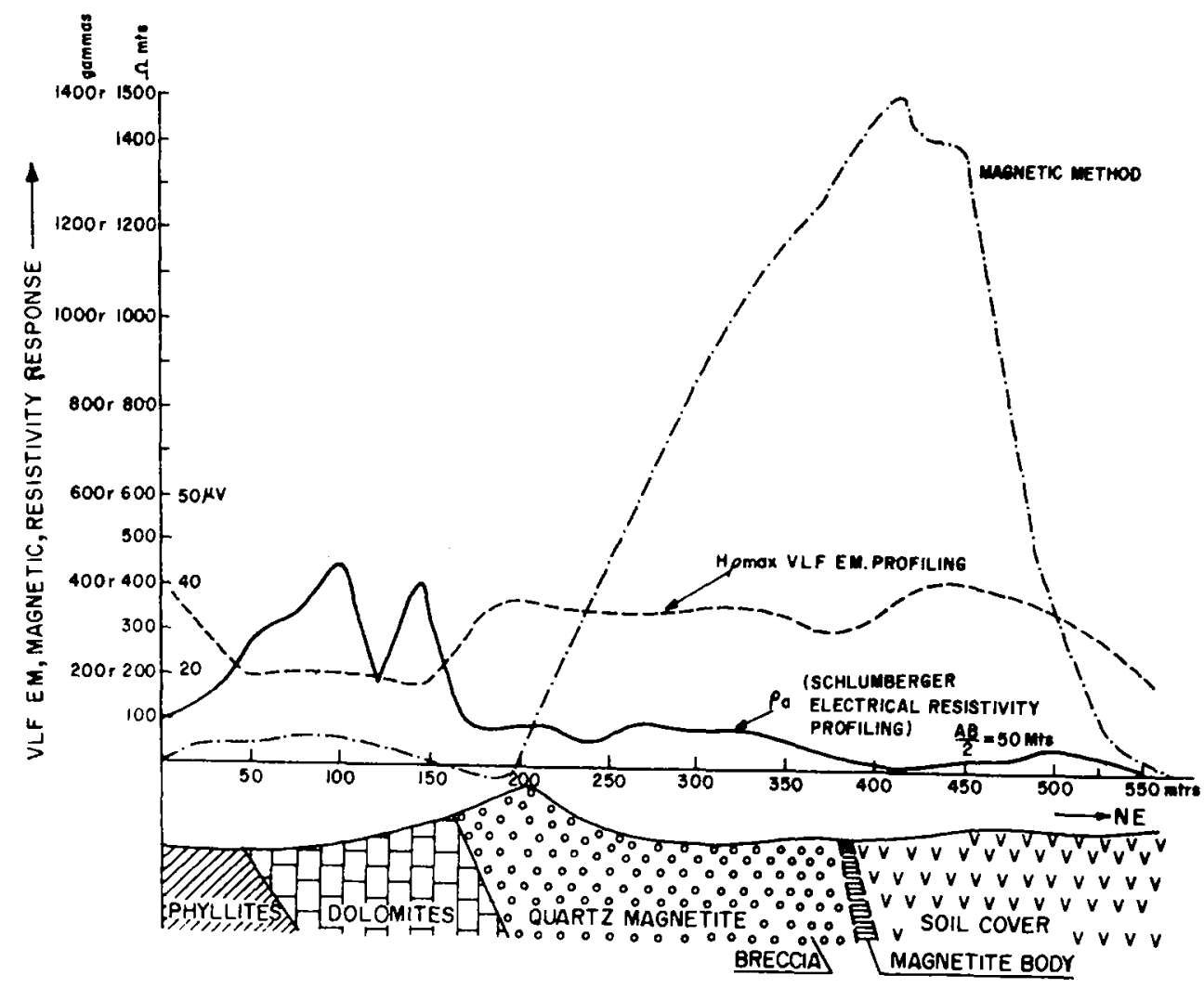

Figure 7. VLF EM Schlumberger resistivity and vertical component magnetic profiles over Shernawala outlier, Khammam. AP.

For estimating the geological mapping capabilities of the VLF equipment the Shernawala outlier, Khammam, AP was selected. In the region metasediments (phyllites, dolomites and quartzites) occurring in a folded pattern have a faulted contact with granites on the western margin and a gradational contact with schists on the eastern side. The presence of faulted breccia and magnetic mineralisation indicated the presence of a nearly NS trending fault within the metasediments (Ramana Rao and Ballurkar 1977; Varaprasada Rao 1977). In figure $7^{*}$ are shown the results of $H \rho_{\max }$ measurements expressed in $\mu \mathrm{V}$. Schlumberger resistivity data with a current electrode separation of $100 \mathrm{~m}$ and potential electrode separation of $4 \mathrm{~m}$, and vertical component magnetic measurements along a SW-NE profile across the Shernawala outlier. It is interesting to note that resistivity data is reflecting the contacts between the phyllites and dolomites and also the contact between dolomites and quartz magnetites, while the magnetic body and the associated structure are not well demarcated by the resistivity profiling. On the other hand the magnetic measurements show a profound anomaly over the magnetic mineralization, while the quartzite-dolomite contact and the dolomite-phyllite contact are not prominently reflected on the profile. The VLF EM profiling data shows a distinct low over relatively resistive dolomites and a steep gradient over the fault structure containing magnetic mineralization. The expected VLF EM responses over the different geological settings are schematically represented in figure 8 (Khmelovskv 1971).

* The magnetic and resistivity data shown in figure 7 was obtained during a field training programme for an UNESCO sponsored short term course in which the first author participated as a teacher. 
(a)

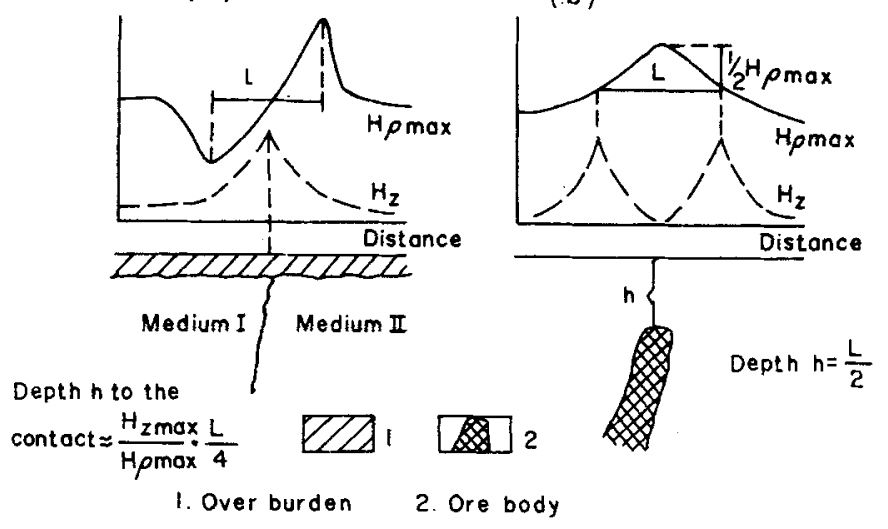

Figure 8. Schematic representation of VI.F EM intensity measurements (after Khmelovsky 19711 .

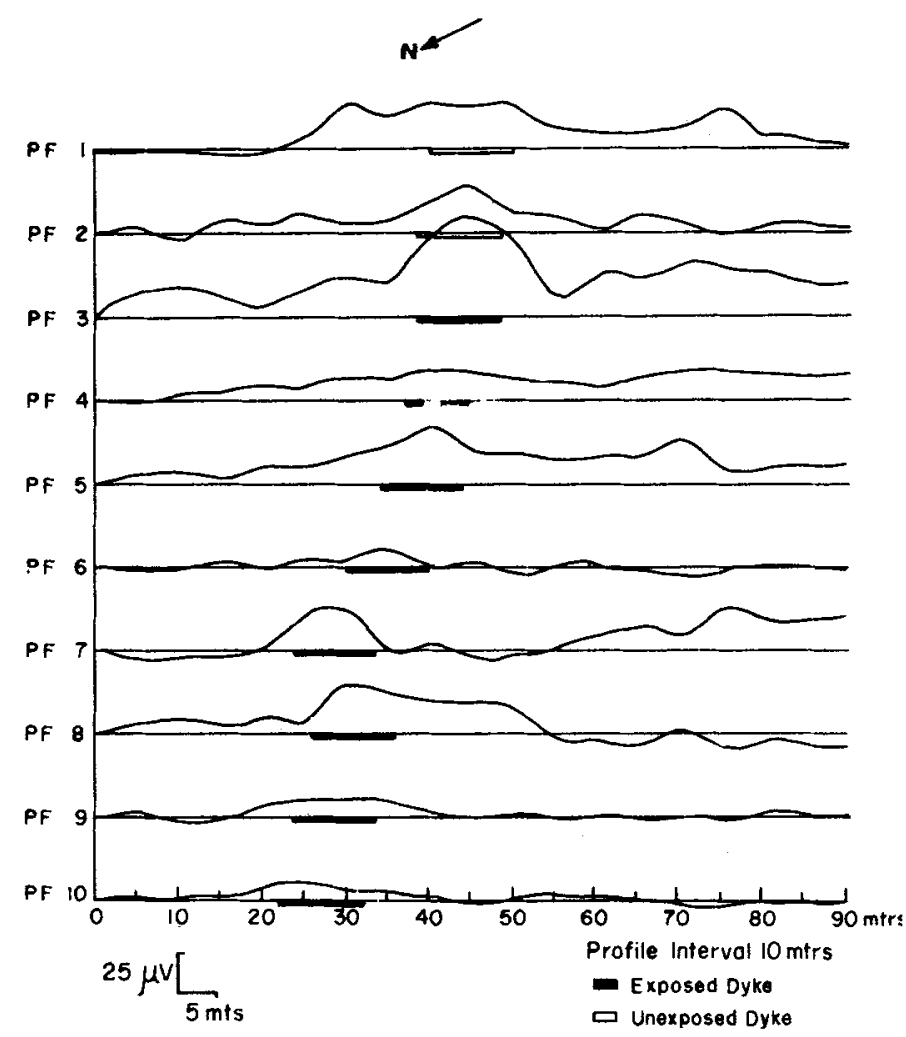

Figure 9. VLF EM results over a dyke near CEG.

The results of VLF EM $\left(H \rho_{\max }\right)$ profiles across a partly exposed dolerite dyke having a strike of $\mathrm{N} 60^{\circ} \mathrm{W}$ in the granitic terrain is shown in figure 9. Ten profiles in $\mathrm{N} 25^{\circ} \mathrm{E}$ direction with a profile interval of $10 \mathrm{~m}$ were covered with the VLF EM method. A distinct anomaly pattern correlatable along all the 10 profiles are observed over the exposed dyke and also over the unexposed parts. 
Usually, when the signal to noise ratio is small or the strike extent is limited to a small number of profiles a visual assessment of the strike direction is not always possible. Under such conditions the cross correlation and stacking techniques (Bhimashankaram et al 1973) can be used for effectively enhancing the signal to noise ratio enabling a reliable detection of the strike. Schaub (1963) proposed the use of a modified correlation $K_{p}^{i, j}$ expressed as a function of the space co-ordinate (Index $p$ ) itself, thereby facilitating the exact spatial location of the expected trends. The correlation function $K_{p}^{i, j}$ can be expressed as

$$
K_{p}^{i, j}=\frac{1}{2 n+1} \quad \sum_{l=-n}^{n} f_{i, p+l} \cdot f_{j, p-l}
$$

By convention the $K_{p}^{i, j}$ function is referred in space to the $p$ th point of a parallel profile midway between profiles $i$ and $j$ and has certain advantages over other correlation methods such as resolution of multiple trends and is capable of distinguishing between correlation of even and odd shaped anomalies by its positive and negative peaks.

The modified correlation function computed (choosing $n=1$ ) for the data presented in figure 9 is shown in figure 10 . It is evident from figure 10 that the computed $K_{p}^{i, j}$ function helps in demarcating the dyke. Such investigations have an application in groundwater exploration in the hard rock terrain.

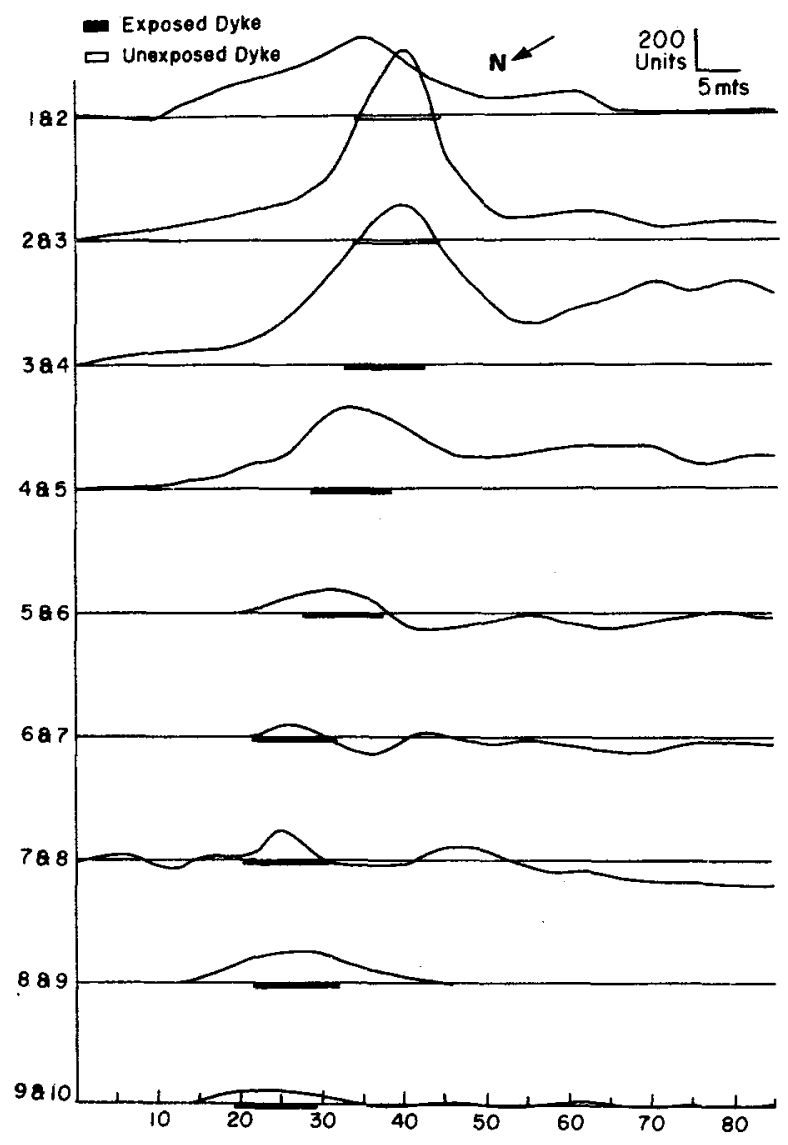

Figure 10. Schaub's modified correlation for VLF data over a dyke near CEG. 


\section{Conclusions}

(a) The VLF EM fields emanating from NWC-Australia can be utilised for fast exploration of fairly resistive ground for (i) geological mapping and (ii) groundwater investigations. These are demonstrated by the field experiments in India utilising the equipment designed and developed by the authors.

(b) Since the diurnal variations are very prominent the intensity measurements in VLF EM prospecting should be properly corrected for the diurnal variations.

\section{Acknowledgements}

The authors express their gratitude to Dr S M Varaprasada Rao, Department of Geophysics. for providing the necessary magnetic data and to the participants of the UNESCO sponsored course for the assistance given in the field.

\section{References}

Bhimasankaram V L S. Tarkhov A G. Nikitin A A and Seshagiri Rao S V S 1973 Geophys. Prospect. 21 464.471

Baker H A and Myers J O 1979 Geoexploration 17 55-63

Baker H A and Myers J O 1980 Geoexploration 18 135-144

Coney D P 1977 Geoexploration 15 19-35

Eherle D 1981 Geoexploration 19 103-114

Fraser D C 1969 Geophysics 34 958-967

Kaikkonen P 1977 J. Geophys. 43 179-192

Khmelovsky V K 197 I Basic course of electrical prospecting (in Russian) Part II (Moscow: University Publication) p. 270

Olsson O 1980 Geophys. Prospect. 28 415-434

Patangay N S 1977 Lectures on Geophysics for Geologists and Engineers (ed.) V L S Bhimasankaram and V K Gaur (Hyderabad: A E G Publication)

Paterson N R and Ronka V 1971 Geoexploration 9 7-26

Poddar M 1981 Pure \& Appl. Geoph!s. 119 1024-1036

Poddar M 1982 Geophysics 47 1059-1067

Ramana Rao N and Ballurkar A 1977 Met. Miner. Rev. 16 10-13

Sacit Saydam A 1981 Geophisics 46 1594-1605

Schauh Yu I 1963 Bull. Izvestia Acad. Sci. USSR Geophysics series No. 42

Sedelnikov E S. Gridchin V M. Gardev S G. Lunin Yu G and Rogachev B V 1971 Method of long wavelength radiokip with SDVR 3 equinment (in Russian) Tchinigri Moscow

Stratton .I A 1941 Electromagnetic theor. (New York: McGraw Hill) p. 587

Sictor BS 1973 Theory: methodology and interpretation of low frequency induction prospecting data (in R(ivian) (Moscow: Nedra) p. 247

latkhil A (i 1961 Basis of Geophisical prospecting bl radiokip method (in Russian) Moscow p. 209

Varaprisada Rao S M 1977 Integrated Geophysical investigations over Shernavala outlier. Khammam distric!. A $P$ Ph.D. Thesis. Osmania University (unpublished) 